\title{
Twenty-six years of enteric fever in Australia: an epidemiological analysis of antibiotic resistance
}

\section{Robert J Commons \\ MB BS, BMedSci, DipObsGyn, \\ Registrar \\ Emma McBryde MB BS, FRACP, PhD Head of Epidemiology \\ Mary Valcanis BSc, MPH, Section Head, Enteri Reference Laboratory ${ }^{2}$ \\ Joan Powling BAgrSC Coordinator National Enteric Pathogens Surveillance Scheme \\ Alan Street \\ MB BS, FRACP, \\ Deputy Directo \\ Geoff Hogg \\ BM, BS, FRACP Director ${ }^{2}$ \\ 1 Victorian Infectious Diseases Service, Royal Melbourne Hospital Melbourne, VIC \\ 2 Microbiological \\ Diagnostic Unit Public \\ Health Laboratory, University of Melbourne Melbourne, VIC. \\ robert.commons@ gmail.com}

MJA 2012; 196: 332-336 doi: $10.5694 / \mathrm{mjal} 2.10082$
E nteric fever is caused by Salmonella enterica subspecies enterica serovar Typhi (S. Typhi) and serovars Paratyphi A and B (S. Paratyphi A and B). Enteric fever is estimated to affect 27 million people annually worldwide, and has an estimated annual mortality of around $216000 .{ }^{1}$ It causes epidemics in Central America, Asia, Africa and the Middle East. ${ }^{2}$ Over the past 20 years, the development of antibiotic resistance has compromised the therapy of this infection.

Chloramphenicol, ampicillin and trimethoprim-sulfamethoxazole had previously been the mainstays of treatment of enteric fever, but resistance to these agents (multidrug resistance) emerged in the late 1980s in the Indian subcontinent. ${ }^{3}$ Quinolone agents, such as ciprofloxacin, initially retained activity against multidrug-resistant (MDR) isolates, but reports of quinolone treatment failure soon appeared, and decreased susceptibility to quinolones is now widespread in the Indian subcontinent and in many Asian countries. ${ }^{2}$

In Australia, enteric fever is an "exotic" disease, and selection of an agent for treatment of this infection must take into account likely patterns of antibiotic resistance in the country of acquisition. The Australian Therapeutic guidelines: antibiotic now recommend that first-line treatment for patients with enteric fever acquired in the Indian subcontinent or SouthEast Asia should be with azithromycin or ceftriaxone. ${ }^{4}$

S. Typhi and S. Paratyphi isolates from patients in all Australian states and territories are sent to the University of Melbourne Microbiological Diagnostic Unit Public Health Laboratory for phage typing and antibiotic susceptibility testing. The entire dataset has not been analysed or reported previously, and published information about antibiotic resistance in Australian $S$. Typhi and $S$. Paratyphi isolates is limited. ${ }^{5}$ We aimed to investigate the

Abstrac

Objectives: To determine incidence and trends in antibiotic resistance in Australian Salmonella enterica subspecies enterica serovars Typhi (S. Typhi) and Paratyphi (S. Paratyphi) isolates over the past 26 years.

Design: A retrospective analysis of consecutive microbiologically confirmed enteric fever isolates.

Participants and setting: All S. Typhi and S. Paratyphi isolates from patients diagnosed with enteric fever in Australia between 1985 and 2010.

Main outcome measures: Incidence and variation in antibiotic resistance over time and according to country of origin.

Results: We analysed 2551 isolates, which originated from 74 countries or regions, mainly India (33\%) and Indonesia (22\%). The incidence among Australian residents increased from four to five before 2003 to seven cases per million person-years after 2003. Multidrug resistance (chloramphenicol, ampicillin, trimethoprim) and nalidixic acid resistance emerged rapidly from the early 1990s, with nalidixic acid resistance increasing to $70 \%$ in 2009-2010, while multidrug resistance was relatively stable at between $4 \%$ and $11 \%$. Nalidixic acid and multidrug resistance rates are highest in isolates from the Indian subcontinent. Some countries in South-East Asia, such as Indonesia, had very low rates of resistance; however, this varied across the region.

Conclusions: Nalidixic acid resistance has become widespread in enteric fever isolates from the Indian subcontinent and some parts of South-East Asia, justifying the use of ceftriaxone or azithromycin rather than ciprofloxacin as first-line treatment. However, resistance in some countries remains rare, potentially allowing treatment to be adjusted according to country of origin.

epidemiology and variations in antibiotic resistance of these isolates over the past 26 years to further inform antibiotic choice.

\section{Methods}

\section{Isolates}

We investigated consecutive isolates of S. Typhi and S. Paratyphi collected in Australia between January 1985 and December 2010. Isolates from patients in all Australian states and territories were collected and processed centrally at the University of Melbourne Microbiological Diagnostic Unit Public Health Laboratory.

Duplicate isolates from the same patient were removed. In addition to submission of isolates, referring laboratories provide data on age, sex, isolation date, isolation site and reported country of origin.

\section{Susceptibility testing}

Susceptibility to antibiotics was determined when isolates were first received using standard methods previously described. ${ }^{6}$ Susceptibility testing was performed by agar dilution and determined using Clinical and Laboratory Standards Institute breakpoint concentrations for resistance ${ }^{7}$ (Box 1). The breakpoint for low-level ciprofloxacin resistance reflects reports of reduced efficacy in some isolates above $0.06 \mathrm{mg} / \mathrm{L}^{8}{ }^{8}$ Susceptibility testing for ciprofloxacin resistance was commenced in January 1994 and for cefotaxime in December 2000.

MDR S. Typhi and S. Paratyphi isolates are defined by resistance to ampicillin, trimethoprim and chloramphenicol. ${ }^{9}$ By convention, nalidixic acid resistance is used as a surrogate for fluoroquinolone resistance; some patients infected with nalidixic-acid resistant (NAR) isolates have been reported to have poor clinical response rates to ciprofloxacin despite the isolates testing as susceptible to ciprofloxacin using breakpoints for Enterobacteriaceae. ${ }^{8}$

\section{Statistical analysis}

The outcome variables of interest were numbers of $S$. Typhi, S. Paratyphi 


\begin{tabular}{lc}
$\begin{array}{l}1 \\
\text { Breakpoint concentrations used to } \\
\text { define antibiotic susceptibility }\end{array}$ \\
Antibiotic & $\begin{array}{c}\text { Breakpoint } \\
\text { concentration }\end{array}$ \\
\hline Ampicillin & $16 \mathrm{mg} / \mathrm{L}$ \\
\hline Chloramphenicol & $16 \mathrm{mg} / \mathrm{L}$ \\
\hline Trimethoprim & $8 \mathrm{mg} / \mathrm{L}$ \\
Nalidixic acid & $16 \mathrm{mg} / \mathrm{L}$ \\
Ciprofloxacin & $>0.06$ to \\
(low-level resistance)* & $<2 \mathrm{mg} / \mathrm{L}$ \\
Ciprofloxacin & $2 \mathrm{mg} / \mathrm{L}$ \\
Cefotaxime & $1 \mathrm{mg} / \mathrm{L}$ \\
\hline
\end{tabular}

*Low-level ciprofloxacin resistance is also referred to as decreased ciprofloxacin susceptibility.

A and S. Paratyphi B isolates (first clinical isolate only) and antibiotic resistance patterns. Predictor variables included age, sex, and year and country of travel. Methods used were exact tests for binary and categorical predictors and binary outcome variables (such as resistance rate by region). Logistic regression analysis was used for continuous predictor variables with binary outcome variables (such as age and resistance rates). Denominator data for population incidence were obtained from the Australian Bureau of Statistics. ${ }^{10}$ Data were analysed using Stata 10 (StataCorp, College Station, Tex, USA).

\section{Results}

\section{Characteristics of patients with enteric fever}

We analysed 2551 isolates, including 1573 S. Typhi isolates, 810 S. Paratyphi A isolates and 168 S. Paratyphi B isolates. There was a statistically significant male predominance among patients with S. Typhi (56.7\%; 95\% CI, $54.2 \%-59.2 \%)$ and S. Paratyphi A (57.6\%; 95\% CI, $54.0 \%-60.9 \%)$ isolates, while $S$. Paratyphi A isolates were generally collected from older patients with a median age of 27 years (interquartile range [IQR], 21-36 years), compared with 24.5 years (IQR, 12-37 years) for $S$. Typhi. Patients ranged in age from 0 to 97 years.

Data about the country or region of origin were available for 1917 isolates $(75 \%)$. There were 74 countries or regions represented, with the most common sources of acquisition being India (33\% of isolates) and Indonesia (22\% of isolates) (Box 2 ).

The incidence of enteric fever cases manifest in Australia was relatively stable during the late 1980s and 1990s at three per million person-years for $S$. Typhi and two per million personyears for $S$. Paratyphi. However, there has been an upsurge since 2003 in $S$. Typhi to around four to five per million person-years and in $S$. Paratyphi to around three per million personyears (Box 3).

\section{Trends in overall resistance}

Before 1991, minimal drug resistance was identified in any enteric fever isolate from Australia (Box 4 and Box 5).

MDR S. Typhi isolates were first detected in Australia from 1989 to 1990 and the rate increased rapidly to $13.7 \%$ by $1991-1992$. Thereafter, the 2 -year incidence has remained relatively stable, varying between $7.1 \%$ and $14.6 \%$ (Box 4). Multidrug resistance has been restricted almost exclusively to $S$. Typhi isolates; there have been only six MDR $S$. Paratyphi isolates, and none since 2001.

2 Origin of enteric fever isolates in Australia, 1985-2010

\begin{tabular}{lcccc} 
& \multicolumn{3}{c}{ Salmonella enterica subspecies enterica serovar, no. (\%) } \\
\cline { 2 - 4 } Country & $\begin{array}{c}\text { S. Typhi, } \\
n=1224\end{array}$ & $\begin{array}{c}\text { S. Paratyphi A, } \\
n=622\end{array}$ & $\begin{array}{c}\text { S. Paratyphi B, } \\
n=71\end{array}$ & $\begin{array}{c}\text { Total, no. (\%) } \\
n=1917\end{array}$ \\
\hline India & $371(30.3 \%)$ & $260(41.8 \%)$ & $4(5.6 \%)$ & $635(33.1 \%)$ \\
\hline Indonesia & $268(21.9 \%)$ & $119(19.1 \%)$ & $31(43.7 \%)$ & $418(21.8 \%)$ \\
\hline Bangladesh & $69(5.6 \%)$ & $45(7.2 \%)$ & 0 & $114(5.9 \%)$ \\
\hline Pakistan & $68(5.6 \%)$ & $37(5.9 \%)$ & 0 & $105(5.5 \%)$ \\
\hline Papua New Guinea & $64(5.2 \%)$ & $1(0.2 \%)$ & 0 & $65(3.3 \%)$ \\
\hline Philippines & $47(3.8 \%)$ & $10(1.6 \%)$ & 0 & $57(3.0 \%)$ \\
\hline Thailand & $29(2.4 \%)$ & $19(3.1 \%)$ & $1(1.4 \%)$ & $49(2.6 \%)$ \\
\hline Cambodia & $16(1.3 \%)$ & $30(4.8 \%)$ & 0 & $46(2.4 \%)$ \\
\hline Lebanon & $41(3.3 \%)$ & $2(0.3 \%)$ & 0 & $43(2.2 \%)$ \\
\hline Nepal & $17(1.4 \%)$ & $24(3.9 \%)$ & 0 & $41(2.1 \%)$
\end{tabular}

Nalidixic acid resistance did not manifest in Australia until 1993-1994, several years after the appearance of MDR isolates. The rate of resistance has climbed steadily since, and by 2009-2010, was identified in $69.8 \%$ of isolates (Box 5). Nalidixic acid resistance increased rapidly in $S$. Paratyphi A until 2005-2006, but has plateaued since $(78.5 \%$, in 2009-2010), whereas resistance continues to increase among S. Typhi isolates $(68.0 \%$ in 2009-2010). There have only been four cases of nalidixic acid resistance in S. Paratyphi B isolates. Two S. Typhi and two $S$. Paratyphi A isolates have demonstrated ciprofloxacin resistance.

Combined multidrug and nalidixic acid resistance was first recognised in 2003; it has been restricted to $S$. Typhi and was identified in $13 \%$ of such isolates in 2007-2008. Of note, 14 S. Typhi isolates and five $S$. Paratyphi isolates demonstrated susceptibility to nalidixic acid and decreased ciprofloxacin susceptibility.

Resistance to other agents remains uncommon. Since testing began 11 years ago, only one isolate ( $S$. Typhi, originating from India in 2009) of 1253 isolates tested has been resistant to third-generation cephalosporins.

\section{Patterns of resistance according to country of origin}

\section{Multidrug resistance}

Among 158 MDR isolates (almost all S. Typhi - see above), country of origin was available for 127 , of which

3 Population-based incidence of Australian enteric fever isolates, $1985-2010$

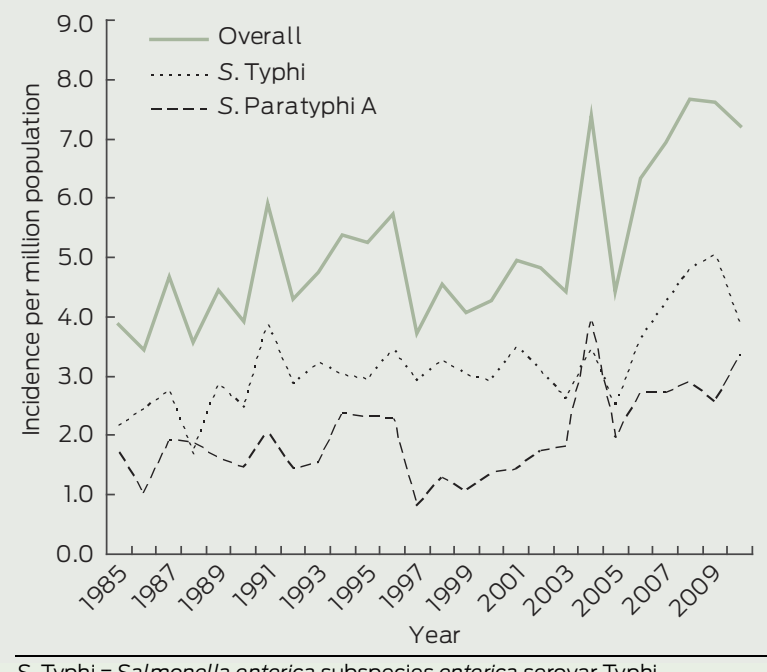

S. Typhi = Salmonella enterica subspecies enterica serovar Typhi. S. Paratyphi = Salmonella enterica subspecies enterica serovar Paratyphi. 


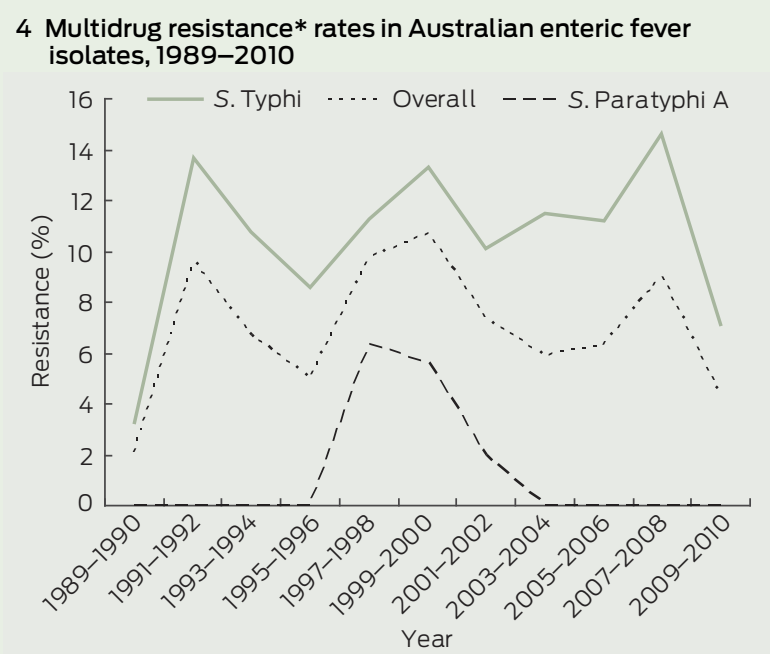

S. Typhi = Salmonella enterica subspecies enterica serovar Typhi. S. Paratyphi = Salmonella enterica subspecies enterica serovar Paratyphi. * Multidrug resistance is defined as resistance to chloramphenicol, ampicillin and trimethoprim.

5 Nalidixic acid resistance rates in Australian enteric fever isolates, 1993-2010*

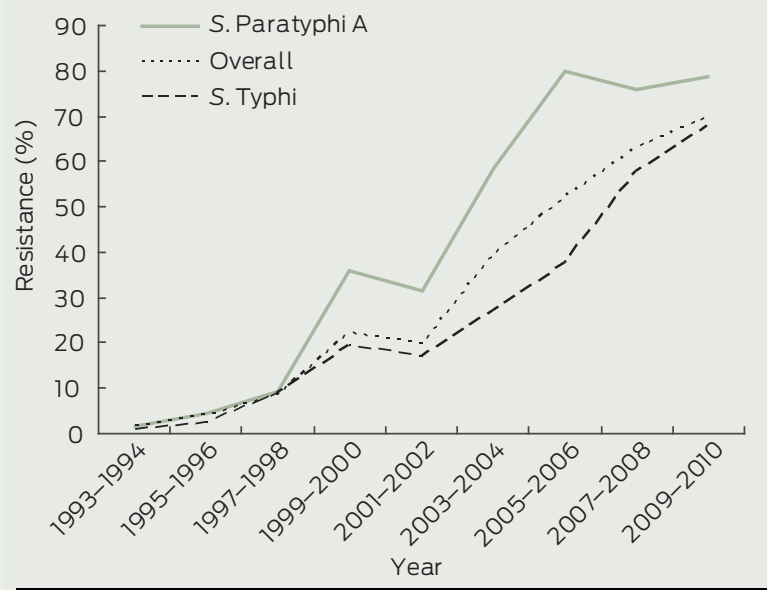

S. Typhi = Salmonella enterica subspecies enterica serovar Typhi. S. Paratyphi = Salmonella enterica subspecies enterica serovar Paratyph * There was one S. Paratyphi A isolate resistant to nalidixic acid detected in 1987-1988.
Salmonella serovar; for example, resistance was $78.8 \%$ among 560 isolates from India (S. Paratyphi, $82.8 \%$; S. Typhi, $76.6 \%$ ), and $33.7 \%$ in isolates from Pakistan (Box 6 and Box 7).

In other countries and regions, nalidixic acid resistance emerged later and rates have been lower and more variable than in the Indian subcontinent (Box 6 and Box 7). In isolates from South-East Asia since 1993, nalidixic acid resistance was more common among S. Paratyphi (8.8\%) than S. Typhi (4.1\%). South-East Asian countries with the highest rates of resistance in $S$. Paratyphi were Vietnam (40.0\%), Burma (33.3\%) and the Philippines $(20.0 \%)$. Countries with the highest rates of nalidixic acid resistance in $S$. Typhi were Cambodia $(28.6 \%)$, Thailand $(23.1 \%)$ and Malaysia (14.3\%). In contrast, the rate of resistance in $S$. Typhi was very low in isolates from Indonesia $(0.5 \%)$ and no resistance was seen in the Pacific region (including Papua New Guinea) or China.

\section{Discussion}

While the incidence of enteric fever manifest in Australia remains low, the frequency of travel to and from regions of high endemicity has led to an increase over recent decades. Our study provides a comprehensive assessment of the incidence of enteric fever over the past 26 years and describes the rapid evolution of multidrug and nalidixic acid resistance perhaps a portent of what might occur with ceftriaxone and azithromycin.

Between 1985 and 2010, we observed an increase in the incidence of $S$. Typhi and S. Paratyphi; from four to five before 2003 to seven cases per million person-years after 2003. This is comparable to increases in the United States, ${ }^{11}$ United Kingdom, ${ }^{12}$ and Canada. ${ }^{13}$ The median age and demographics likely reflect the population travelling to and from countries where enteric fever is endemic.

The Indian subcontinent has seen a rapid increase in nalidixic acid resistance, first (in the early 1990s) in S. Paratyphi A and later (since 2000) in $S$. Typhi. For both serovars, resistance was first recognised in India and was subsequently reported in the rest of the subcontinent over the following 5 years. The emergence of nalidixic acid resistance across the Indian subcontinent in returned travellers from this study reflects rates found within country-specific studies from India, ${ }^{14}$ Bangladesh ${ }^{15}$ and Nepal. ${ }^{16}$ Resistance is thought to have developed rapidly due to excessive and unregulated antibiotic use in these developing countries.

Resistance rates in South-East Asia are more regionally heterogeneous and country-specific. S. Paratyphi A isolates have demonstrated nalidixic acid resistance in this region since 2003, and there is a trend of increasing resistance across the region. However, resistance remains rare in some countries, including Indonesia and Cambodia. This contrasts with $S$. Typhi isolates in South-East Asia, where there are generally low rates of nalidixic acid and multidrug resistance. Interestingly, with S. Typhi, Cambodia stands out as having higher nalidixic acid resistance (25\%) and multidrug resistance $(38 \%)$ rates than its neighbours. This finding is in keeping with another study of 41 blood culture isolates of $S$. Typhi from 2006 to 2009 conducted in Cambo$\mathrm{dia}^{17}$ in which multidrug resistance was found in $56 \%$ of isolates and nalidixic acid resistance in $81 \%$.

Our finding of very low rates of antibiotic resistance in Indonesia for both $S$. Typhi and S. Paratyphi differs from an Indonesian study, which showed rates of multidrug resistance of up to $6.83 \%$ and ciprofloxacin resistance of $3.90 \%$ from South Sulawesi. ${ }^{18}$ This discrepancy is likely explained by a predominance of isolates in our study arising from Bali, the most popular Australian tourist destination in Indonesia.

In our study, the rate of nalidixic acid resistance in $S$. Typhi isolates after 2001 was $45 \%$. This compares to $38 \%$ of isolates between 1999 and 2006 in the US, ${ }^{19} 56 \%$ of isolates between 2000 and 2006 in Canada ${ }^{13}$ and $60 \%$ of isolates between 2000 and 2006 from the UK. ${ }^{20}$

A combination of susceptibility to nalidixic acid and reduced susceptibility to ciprofloxacin was seen in 19 isolates in this study. This is likely due to transmission of isolates with plasmid-mediated reduced susceptibility 
6 Rates of nalidixic acid and multidrug resistance in S. Typhi and S. Paratyphi A isolates

\begin{tabular}{|c|c|c|c|c|c|c|}
\hline \multirow[b]{2}{*}{ Country } & \multicolumn{2}{|c|}{ Since 1989} & \multicolumn{4}{|c|}{ Since 1993} \\
\hline & $\begin{array}{l}\text { S. Typhi } \\
\text { isolates } \\
\text { tested for } \\
\text { MDR }\end{array}$ & $\begin{array}{c}\text { S. Typhi } \\
\text { MDR rate, } \\
\%\end{array}$ & $\begin{array}{l}\text { S. Typhi } \\
\text { isolates } \\
\text { tested } \\
\text { for NAR }\end{array}$ & $\begin{array}{c}\text { S. Typhi } \\
\text { NAR rate, } \\
\%\end{array}$ & $\begin{array}{l}\text { S. Paratyphi A } \\
\text { isolates } \\
\text { tested for } \\
\text { NAR }\end{array}$ & $\begin{array}{c}\text { S. Paratyphi } \\
\text { A NAR rate, } \\
\%\end{array}$ \\
\hline $\begin{array}{l}\text { Indian } \\
\text { subcontinent }\end{array}$ & 512 & $19.1 \%$ & 477 & $65.6 \%$ & 327 & $77.7 \%$ \\
\hline India & 354 & $14.7 \%$ & 329 & $76.6 \%$ & 227 & $82.8 \%$ \\
\hline Sri Lanka & 6 & $16.7 \%$ & 6 & $33.3 \%$ & 9 & $100.0 \%$ \\
\hline Bangladesh & 69 & $30.4 \%$ & 67 & $55.2 \%$ & 41 & $73.2 \%$ \\
\hline Nepal & 15 & $6.7 \%$ & 15 & $26.7 \%$ & 21 & $71.4 \%$ \\
\hline Pakistan & 68 & $33.8 \%$ & 60 & $30.0 \%$ & 29 & $41.4 \%$ \\
\hline South-East Asia & 355 & $4.2 \%$ & 318 & $4.1 \%$ & 181 & $8.8 \%$ \\
\hline Burma & 6 & $16.7 \%$ & 6 & 0 & 9 & $33.3 \%$ \\
\hline Vietnam & 23 & $17.3 \%$ & 17 & $11.8 \%$ & 5 & $40.0 \%$ \\
\hline Philippines & 41 & 0 & 26 & 0 & 5 & $20.0 \%$ \\
\hline Thailand & 28 & $7.1 \%$ & 13 & $23.1 \%$ & 10 & $10.0 \%$ \\
\hline Malaysia & 9 & 0 & 7 & $14.3 \%$ & 5 & $40.0 \%$ \\
\hline Indonesia & 248 & $0.8 \%$ & 221 & $0.5 \%$ & 106 & $3.8 \%$ \\
\hline Cambodia & 15 & $40.0 \%$ & 14 & $28.6 \%$ & 28 & $3.6 \%$ \\
\hline $\begin{array}{l}\text { South and } \\
\text { Central America }\end{array}$ & 16 & 0 & 11 & 0 & 1 & $100.0 \%$ \\
\hline China & 20 & $5.0 \%$ & 16 & 0 & 4 & $75.0 \%$ \\
\hline Europe & 14 & 0 & 12 & 0 & 0 & 0 \\
\hline Middle East & 44 & $9.1 \%$ & 32 & $6.3 \%$ & 5 & 0 \\
\hline Pacific Region & 101 & $1.0 \%$ & 89 & 0 & 4 & $50.0 \%$ \\
\hline $\begin{array}{l}\text { Sub-Saharan } \\
\text { Africa }\end{array}$ & 28 & $10.7 \%$ & 26 & 0 & 3 & $33.3 \%$ \\
\hline
\end{tabular}

to ciprofloxacin that does not affect nalidixic acid. ${ }^{7}$ Similar isolates with reduced ciprofloxacin susceptibility have been associated with treatment failure, ${ }^{7,21}$ and clinicians should be aware that nalidixic acid testing alone may no longer indicate fluoroquinolone susceptibility.

Therapeutic options for treatment of MDR and NAR enteric fever are limited to ceftriaxone and azithromycin, so emergence of resistance to either of these agents would be a major concern. Only one isolate in this study tested resistant to ceftriaxone, which supports the observation that resistance to third-generation cephalosporins is yet to become established. ${ }^{13}$ Our reference laboratory does not routinely perform azithromycin resistance testing at present because minimum inhibitory concentration (MIC) cut-offs have not been determined. However, there have already been reports of enteric fever isolates with high azithromycin MICs associated with clinical failure, ${ }^{22}$ so this situation will require careful monitoring. Gatifloxacin, an extendedspectrum fluoroquinolone with good clinical activity against $S$. Typhi and $S$. Paratyphi, including NAR isolates, has unfortunately been withdrawn from the market in many countries due to reports of an association with hypoglycaemia and hyperglycaemia. ${ }^{23}$

Our study did not record whether travellers were visiting friends or relatives (VFRs). Previous studies have demonstrated an increased risk of enteric fever in such populations, ${ }^{24,25}$ partly due to reduced uptake of pretravel medical advice, including vaccination. It would be useful to be able to ascertain the proportion of VFRs to other tourists who contract enteric fever to determine individual risk and better inform the need for specific population-directed education.

Our study details the emergence of multidrug and nalidixic acid resistance in returned travellers from Australia and others who have acquired enteric fever overseas over the past 26 years. While the rate of multidrug resistance has plateaued, the rate of nalidixic acid resistance has continued to increase in both $S$. Typhi and $S$. Paratyphi isolates. The current Australian Therapeutic guidelines: antibiotic recommend the use of ceftriaxone or azithromycin, ${ }^{4}$ either of which is effective treatment for patients with confirmed or presumptive enteric fever, including drug-resistant infection; however, those who acquire enteric fever in places such as Bali, Indonesia, could be confidently treated with ciprofloxacin due to the low rates of resistance. To ensure emerging resistance is detected and to inform treatment, it is vital that the current comprehensive testing of antibiotic resistance in enteric fever isolates continues.

Acknowledgements: We acknowledge the Australian laboratories that provided isolates for this study and the Victorian Government Department of Health for funding the Microbiological Diagnostic Unit Public Health Laboratory.

Competing interests: No relevant disclosures.

Received 12 Jan 2012, accepted 19 Feb 2012

1 Crump JA, Luby SP, Mintz ED. The global burden of typhoid fever. Bull World Health Organ 2004; 82: 346-353.

2 Parry CM. Typhoid fever. Curr Infect Dis Rep 2004; 6: 27-33.

7 Nalidixic acid resistance rates in Australian enteric fever isolates acquired in the Indian subcontinent and SouthEast Asia, 1993-2010

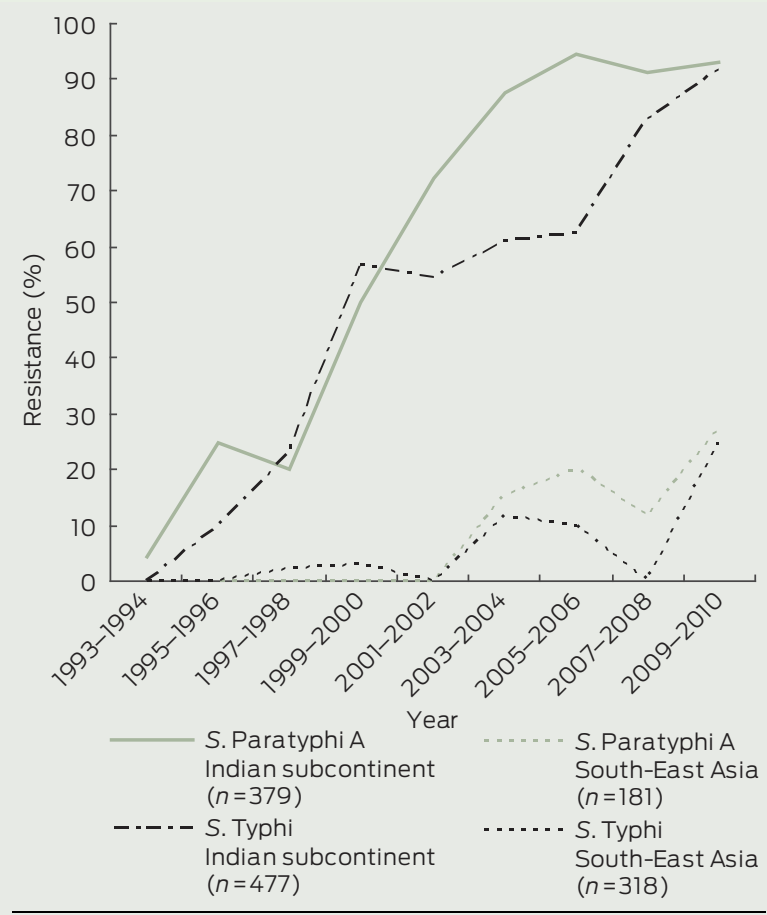

S. Typhi = Salmonella enterica subspecies enterica serovar Typhi. S. Paratyphi = Salmonella enterica subspecies enterica serovar Paratyphi. 
3 Anand AC, Kataria VK, Singh W, et al. Epidemic multiresistant enteric fever in eastern India. Lancet 1990; 335: 352

4 Antibiotic expert group. Therapeutic guidelines: antibiotic. Version 14. Melbourne: Therapeutic Guidelines Limited, 2010.

5 Hume S, Schulz T, Vinton P, et al. Increasing rates and clinical consequences of nalidixic acidresistant isolates causing enteric fever in returned travellers: an 18-year experience. Eur J Clin Microbiol Infect Dis 2009; 28: 963-970.

6 Clinical and Laboratory Standards Institute. Methods for dilution antimicrobial susceptibility tests for bacteria that grow aerobically; Approved standard - eighth edition. Wayne, Pa: CLSI, 2009. (CLSI document M07-A8.)

7 Clinical and Laboratory Standards Institute. Performance standards for antimicrobial susceptibility testing; twenty-first informational supplement. Wayne, Pa: CLSI, 2011. (CLSI document M100-S21.)

8 RoweB, Ward LR, Threlfall EJ. Multidrug-resistant Salmonella Typhi: a worldwide epidemic. Clin Infect Dis 1997; 24: S106-S109.

9 Crump JA, Kretsinger K, Gay K, et al. Clinical response and outcome of infection with Salmonella enterica serotype Typhi with decreased susceptibility to fluoroquinolones: a United States foodnet multicenter retrospective cohort study. Antimicrob Agents Chemother 2008; 52: 1278-1284.

10 Australian Bureau of Statistics. Australian historical population statistics, 2008. http://
www.abs.gov.au/AUSSTATS/abs@.nsf/ DetailsPage/3105.0.65.0012008?0pen Document (accessed Nov 2011).

11 Centers for Disease Control and Prevention (CDC). Notifiable diseases and mortality tables. MMWR Morb Mortal Wkly Rep 2010; 59: 168-181.

12 United Kingdom Health Protection Agency. Salmonella by serotype. 2011. http:// www.hpa.org.uk/Topics/InfectiousDiseases/ InfectionsAZ/Salmonella/EpidemiologicalData/ salmDataHuman (accessed Oct 2011).

13 Demczuk WH, Finley R, Nadon C, et al. Characterization of antimicrobial resistance, molecular and phage types of Salmonella enterica serovar Typhi isolations. Epidemiol Infect 2010; 138: 1414-1426.

14 Chitnis S, Chitnis V, Hemvani N, et al. Ciprofloxacin therapy for typhoid fever needs reconsideration. J Infect Chemother 2006; 12: 402-404.

15 Rahman MM, Haq JA, Morshed MA, et al. Salmonella enterica serovar Typhi with decreased susceptibility to ciprofloxacin-an emerging problem in Bangladesh. Int J Antimicrob Agents 2005; 25: 345-346.

16 Shirakawa T, Acharya B, Kinoshita S, et al. Decreased susceptibility to fluoroquinolones and gyrA gene mutation in the Salmonella enterica serovar Typhi and Paratyphi A isolated in Katmandu, Nepal, in 2003. Diagn Microbiol Infect Dis 2006; 54: 299-303.

17 Kasper MR, Sokhal B, Blair PJ, et al. Emergence of multidrug-resistant Salmonella enterica serovar
Typhi with reduced susceptibility to fluoroquinolones in Cambodia. Diagn Microbio Infect Dis 2010; 66: 207-209.

18 Hatta M, Ratnawati. Enteric fever in endemic areas of Indonesia: an increasing problem of resistance. J Infect Dev Ctries 2008; 2: 279-282.

19 Lynch MF, Blanton EM, Bulens S, et al. Typhoid fever in the United States, 1999-2006. JAMA 2009; 302: 859-865.

20 Threlfall EJ, de Pinna E, Day M, et al. Alternatives to ciprofloxacin use for enteric fever, United Kingdom. Emerg Infect Dis 2008; 14: 860-861.

21 Parry CM, Vinh H, Chinh NT, et al. The influence of reduced susceptibility to fluoroquinolones in Salmonella enterica serovar Typhi on the clinical response to ofloxacin therapy. PLoS Negl Trop Dis 2011; 5: el163.

22 Molloy A, Nair S, Cooke FJ, et al. First report of Salmonella enterica serotype Paratyphi A azithromycin resistance leading to treatment failure. J Clin Microbiol 2010; 48: 4655-4657.

23 Park-Wyllie LY, Juurlink DN, Kopp A, et al. Outpatient gatifloxacin therapy and dysglycemia in older adults. NEngl J Med 2006; 354: 1352-1361.

24 Angell SY, Cetron MS. Health disparities among travellers visiting friends and relatives abroad. Ann Intern Med 2005; 142: 67-72.

25 Nguyen TO, Reddy V, Sahl S, et al. Importance of travel in domestically acquired typhoid fever infections: opportunities for prevention and early detection. J Immigr Minor Health 2009; 11: 139-142. 